\title{
Unmanned Farm utilizes Virtual Fence Technology for Animal Tracking
}

\author{
Jung Kyu Park, Eun Young Park, Jaeho Kim
}

\begin{abstract}
In order to graze animals on farms in large areas, fences must be installed. For this reason, installation and maintenance costs are high. To solve this problem and to manage animals efficiently, we want to use virtual fences based on IoT system. A virtual fence is not about installing a physically contiguous fence but using the least IoT device to get the effect of the existing fence. As described above, since the virtual fence is not an invisible and continuous object, it can be formed in various shapes other than a rectangular shape. Virtual fences can be implemented using a variety of sensors and embedded systems currently available in the marketplace. This can reduce the cost of installing and maintaining an existing fence and provide additional benefits such as tracking the health of the animal and movement tracking. In this paper, we propose a virtual fence algorithm. The proposed algorithm can propose an animal's range of motion and take action according to specified rules. The simulation results show that the proposed algorithm can manage the animals in the virtual fence well.
\end{abstract}

Keywords : Animal tracking, GPS, IoT, Virtual fence

\section{INTRODUCTION}

In the livestock industry, the easiest way to manage animals within a certain range is to build and build a fence. However, the construction of the fence has a problem that the initial cost increases greatly depending on the size of the management area, and manpower and cost for maintenance are large. For this reason, many livestock industries are interested in introducing virtual fence technology using embedded systems [1]. Virtual fence technology utilizes IT technology to create virtual boundaries with functions such as normal fences in an animal environment. Virtual fences are aimed at creating specific areas in large areas and allowing animals to stay out of the area. Compared to regular fences, virtual fences can have several advantages. First, by using IT technology in a virtual fence, the cost of maintaining the existing fence can be reduced. Once the existing fence is built, it costs a lot to move to the new area. However, a virtual fence can cost a lot by moving only a few pieces of equipment. Secondly, introducing a virtual fence enables efficient management of animals. In the environment where the general fence is installed, management of the animal

Revised Manuscript Received on December 30, 2019.

* Correspondence Author

Jung Kyu Park, Dept. of Computer Software Engineering, Changshin University, Changwon-si, Korea. Email: smartjkpark@gmail.com

Eun Young Park, Department of Biomedical Laboratory Science, Shinhan University, Uijeongbu-si, Korea. Email: 71eypark@gmail.com

Jaeho Kim, Department of Electrical and Computer Engineering, Virginia Tech, VA, USA. Email: kjhnet@gmail.com

(C) The Authors. Published by Blue Eyes Intelligence Engineering and Sciences Publication (BEIESP). This is an open access article under the CC BY-NC-ND license (http://creativecommons.org/licenses/by-nc-nd/4.0/) management personnel is needed from time to time, and when the animal leaves the fence, it is difficult to find the animal. With the introduction of virtual fences, animals can be found easily even if they are located in an invisible area [2]. To build a virtual fence, you can use two technologies that are commonly used in IT. The first is to use a radio frequency (RF) signal. In the initial virtual fence study, the $\mathrm{RF}$ module was mentioned and early experiments using it were done. The virtual fence system using the RF module is equipped with a simple system equipped with an RF module for all the animals in the virtual fence, which sends a signal when the animal is moving, processes the signal [2, 3]. The second is to use the GPS module. The GPS module was initially used to track the location of the animals inside the fence [3]. The initial GPS module was unable to accurately measure the position of the animal due to the limitations of technology. Recently, it has been possible to reduce the accuracy to within $\pm 5 \sim 10 \mathrm{~m}$. However, location measurement using GPS can degrade the accuracy of position measurement depending on the terrain environment. In recent years, due to the accurate GPS module, many network devices are adopting GPS module [4, 5, 6, 7]. In this study, we propose a method to implement an unmanned farm using a small embedded board that can fire GPS modules. The unmanned farm can be configured in the form of a polygon and the shape can be modified according to the environmental tax in which the virtual fence is installed. The GPS module and embedded board are built into the necklace and various types of animals to be placed inside the virtual fence. The mounted board must have network capability and transmit to the base station that can collect the received GPS information. Based on the transmitted information, the animals approaching the virtual fence receive a signal from the base station to reproduce sounds that the animal dislikes but can drive the vibration device to control the virtual fence. We prove that the proposed unmanned farm can be implemented through simulation experiments. The rest of the paper is as follows. The following two sections describe the unmanned farm implementation proposed in this paper. Section 3 explains the experimental results. Finally, Section 4 describes conclusions and future plans.

\section{UNMANNED FARM USING VIRTUAL FENCE}

The proposed unmanned fam is consisting with a popular GPS module and an embedded board. At this time, the embedded board should be able to acquire the position information through the GPS module and transmit the information to the base station that can collect GPS information through the network and handle the work. 


\section{Unmanned Farm utilizes Virtual Fence Technology for Animal Tracking}

In recent years, many smart phones have built-in GPS module and network, so it is possible to replace embedded board with smart phone.

Fig. 1 shows the structure of the virtual fence in the simplest way. It is prevalent. Virtual Fence Line (VFL) may be formed in a circular shape having a radius $\mathrm{R}$ from the center point $\mathrm{CP}$ of the fence as a starting point. This is the structure of the most basic virtual fence. Depending on the farm environment, multiple CPs can be arranged to form virtual fences with polygons.

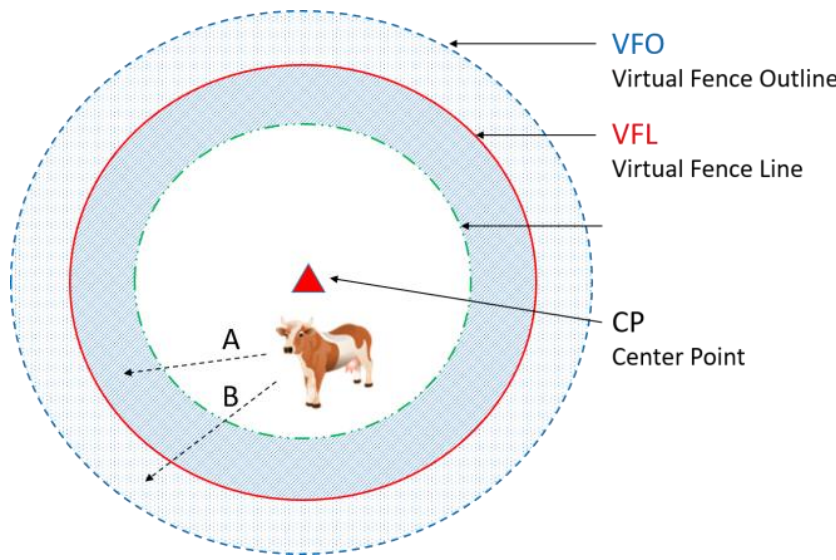

Fig. 1.Structure of Virtual Fence

A certain area can be allocated inside the VFL and this area can be displayed as a virtual fence internal line VFI. If the animal with the GPS is near the VFI, it will play the sound that the animal dislikes, allowing the animal to stay inside the virtual fence. When the animal enters the VFI and approaches the VFL, use a physical device, such as vibration, along with the sound to prevent the animal from escaping the VFL. Finally, if the animal moves out of the VFL and is positioned between the VFOs, it will generate sound and vibration, and will also advise the administrator so that the animal will be prevented from escaping the fence as much as possible. The virtual fence algorithm can be operated based on the rules using the virtual fence structure and the animal location $\mathrm{Ax}$ described above. Table 1 summarizes the virtual fence algorithm rules.

\begin{tabular}{|c|c|}
\hline \multicolumn{2}{|c|}{ Table- I: Rules for warning } \\
\hline $\begin{array}{l}\text { Position of } \\
\text { Animal }\end{array}$ & Action \\
\hline $\mathrm{Ax} \leq(\mathrm{VFI}-\Delta)$ & None \\
\hline$(\mathrm{VFI}-\Delta)<\mathrm{Ax}$ & Play Sound \\
\hline $\mathrm{VFI} \leq \mathrm{Ax} \leq \mathrm{VFL}$ & Play Sound, Vibration \\
\hline $\mathrm{VFL} \leq \mathrm{Ax} \leq \mathrm{VFO}$ & $\begin{array}{l}\text { Play Sound, Vibration, Notify the } \\
\text { administrator }\end{array}$ \\
\hline
\end{tabular}

\section{EXPEIRMENT RESULTS}

In order to verify the proposed unmanned farm algorithm, a simulator program was developed and tested. Fig. 2 shows the simulator produced, and it is possible to construct various types of virtual fences and experiment with various animals. In the experiment, the diameter of the CFL is set to $10 \mathrm{~m}$ assuming a circular virtual fence. The CFI diameter is set to $9 \mathrm{~m}$ and the CFO diameter is set to $11 \mathrm{~m}$. If a circular fence is not possible due to an obstacle in an environment in which a virtual fence is required, a plurality of circular virtual fences may be configured to form a polygon. In addition, a rectangular virtual fence can be constructed.

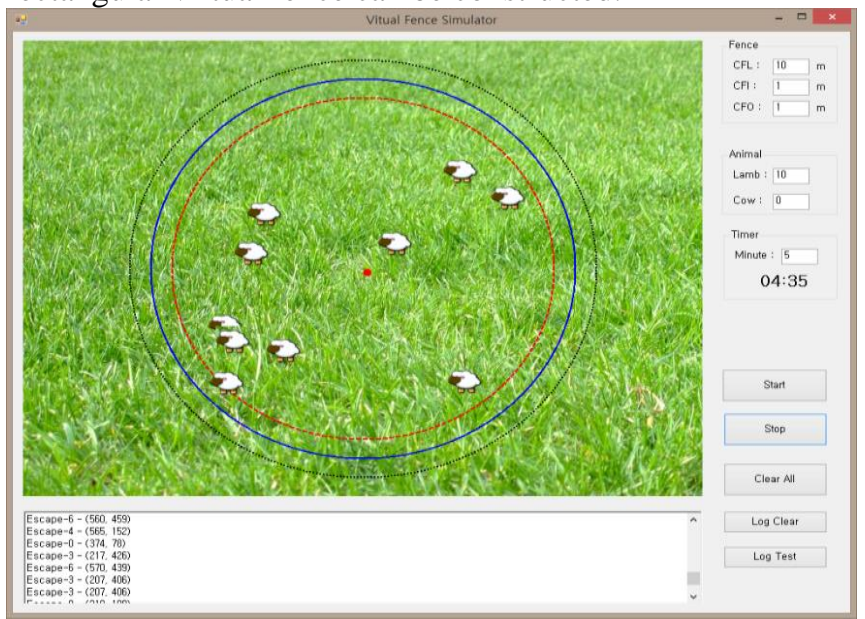

Fig. 2.Simulation program for virtual fence

In the simulation experiment environment, various animals can be experimented. The moving speed of the quantity used in the experiment was set $30 \%$ faster than the positive average speed of $40 \mathrm{~km} / \mathrm{h}$, and the positive shift was randomly set according to the positive habit. It assumes that it has an embedded board equipped with ASCEN ultra miniature GPS module AKML3C (position error $2.5 \sim 3 \mathrm{~m}$ ) and transmits its position once every 1s. At this time, any board can be used if the embedded board can have a GPS module and a network module capable of transmitting GPS data.

Fig. 3 shows the number of attempts to escape beyond the VFL when the experiment was run for 30 minutes with 10 sheep. The number of attempts to escape the fence was 36 times in 8 times and the number of attempted escapes was 7 times in 10 times. Fig. 4 shows the sheep movements at this time. According to the number of escape attempts by virtual fence, the number of escapes decreased by about $42 \%$ when the virtual fence was turned on. This is because the amount of sound and vibration that occurs when the amount exceeds the VFL changes its position to the inside of the VFL.

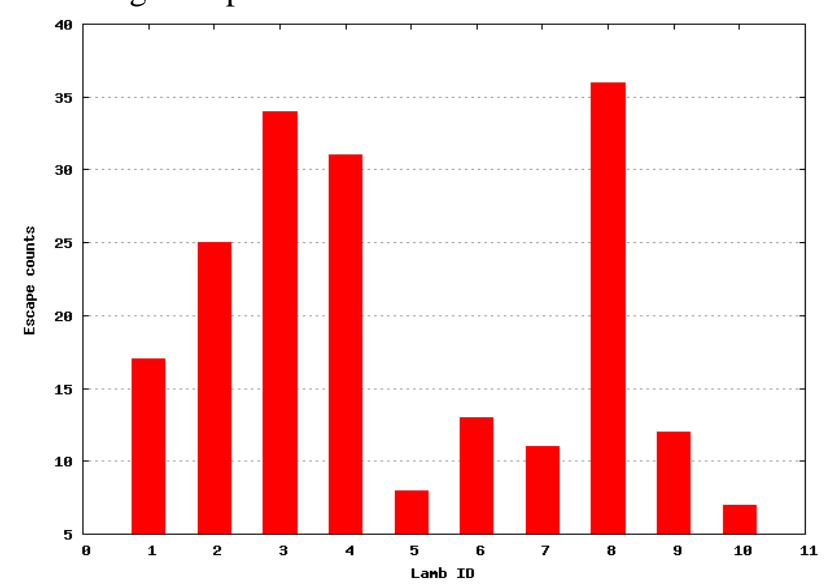

Fig. 3.Number of attempts

Published By:

Blue Eyes Intelligence Engineering \& Sciences Publication 


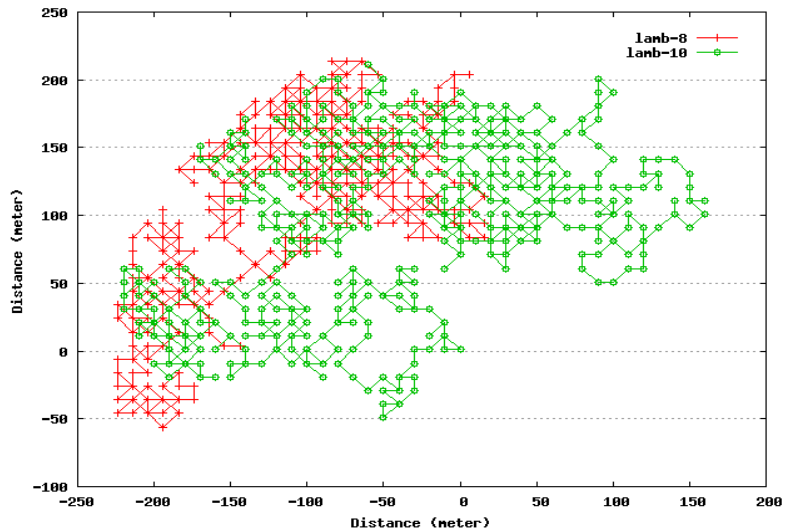

Fig. 4.Posision tracking for the lambs

\section{CONCLUSIONS}

In this paper, we propose a virtual fence and proposed an unmanned farm. A virtual fence is built using IoT, not a physically connected fence. By using this, it is possible to manage the animals efficiently compared to the existing fence, and it is possible to reduce the hand loss of the person. In addition, we propose an algorithm that can control the movement of an animal using an embedded system and show the possibility of the algorithm through simulation experiment. In the future research, we will apply the algorithm and experiment with actual animal IoT equipment. All experiments using animals will be carried out with national permission.

\section{ACKNOWLEDGMENT}

This research was supported by Basic Science Research Program through the National Research Foundation of Korea(NRF) funded by the Ministry of Education (No. 2018R1C1B5046282). This work was supported by Changshin University Research Fund of 2019(Changshin-2019-32)

\section{REFERENCES}

1. J. Chen, T. Tseng, C. Lai, and S. Hsieh, "An Intelligent Virtual Fence Security System for the Detection of People Invading," in Proc. of the 9th International Conference on Ubiquitous Intelligence and Computing and 9th Inter. Conf. on Autonomic and Trusted Computing, 2012 Sep. pp. 786-791.

2. S. Kim, D. Kim, and H. Park, "Animal Situation Tracking Service Using RFID, GPS, and Sensors," in Proc. of the 2010 Second Inter. Conf. on Computer and Network Technology, 2010 Apr. pp. 153-156.

3. V. M. Anu, M. I. Deepika, and L. M. Gladance, "Animal identification and data management using RFID technology," in Proc of the Inter. Conf. on Innovation Information in Computing Technologies, 2015 Feb. pp. 1-6.

4. L. Tang, P. Abplanalp, "GPS guided farm mapping and waypoint tracking mobile robotic system," in Proc of the 2014 9th IEEE Conf. on Industrial Electronics and Applications, 2014 Jun. pp. 1676-1681.

5. S. Koompairojn, C. Puitrakul, T. Bangkok, N. Riyagoon and S. Ruengittinun, "Smart tag tracking for livestock farming," in Proc of the 2017 10th International Conference on Ubi-media Computing and Workshops (Ubi-Media), 2017 pp. 1-4.

6. H. T. Chan, T. A. Rahman, and A. Arsad, "Performance study of virtual fence unit using Wireless Sensor Network in IoT environment," in Proc. of the 2014 20th IEEE International Conference on Parallel and Distributed Systems (ICPADS), 2014 Dec. pp. 873-875.

7. L. Nóbrega, A. Tavares, A. Cardoso, and P. Gonçalves, "Animal monitoring based on IoT technologies," in Proc. of the 2018 IoT Vertical and Topical Summit on Agriculture - Tuscany (IOT Tuscany). 2018 May pp. 1-5.

\section{AUTHORS PROFILE}

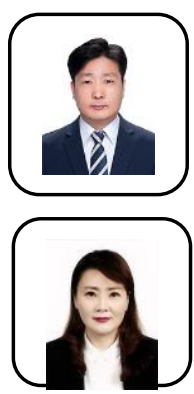

Jung Kyu Park Assistant Professor, Department of Computer Software Engineering, Changshin University, Korea

Eun Young Park Research Professor, Department of Biomedical Laboratory Science, Shinhan University, Korea

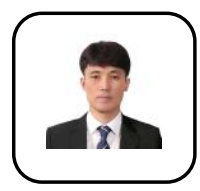

Jaeho Kim Department of Electrical and Computer Engineering, Virginia Tech, VA, USA. 